\title{
New results on the helium stars in the galactic center using BEAR spectro-imagery
}

\author{
T. Paumard ${ }^{1}$, J. P. Maillard ${ }^{1, \star}$, M. Morris ${ }^{2}$, and F. Rigaut ${ }^{3, \star}$ \\ 1 Institut d'Astrophysique de Paris (CNRS), 98b Bd. Arago, 75014 Paris, France \\ 2 University of California, Los Angeles, Div. of Astronomy, Dept. of Physics and Astronomy, Los Angeles, \\ CA 90095-1562, USA \\ 3 Gemini North Headquarter, Hilo, HI 96720, USA
}

Received 22 June 2000 / Accepted 17 October 2000

\begin{abstract}
Integral field spectroscopy of the central parsec of the Galactic Center was obtained at $2.06 \mu \mathrm{m}$ using BEAR, an imaging Fourier Transform Spectrometer, at a spectral resolution of $74 \mathrm{~km} \mathrm{~s}^{-1}$. Sixteen stars were confirmed as "helium stars" by detecting the HeI $2.058 \mu \mathrm{m}$ line in emission, providing a homogeneous set of fully resolved line profiles. These observations allow us to discard some of the earlier detections of such stars in the central cluster and to add three new stars. The sources detected in the BEAR data were compared with adaptive optics images in the $K$ band to determine whether the emission was due to single stars. Two sub-classes of almost equal number are clearly identified from the width of their line profiles, and from the brightness of their continuum. The first class is characterized by very broad line profiles $\left(F W H M \simeq 1000 \mathrm{~km} \mathrm{~s}^{-1}\right)$ and by their relative faintness. The other, brighter in $K$ by an average factor of $\sim 9$, has a much narrower emission component of width $\simeq 200 \mathrm{~km} \mathrm{~s}^{-1}$. Most of the emission lines show a P Cygni profile. From these results, we propose that the latter group is formed of stars in or near the LBV phase, and the other one of stars at the WR stage. The division into two groups is also shown by their spatial distribution, with the narrow-line stars in a compact central cluster (IRS 16) and the other group distributed at the periphery of the central cluster of hot stars. In the same data cube, streamers of interstellar helium gas are also detected. The helium emission traces the densest parts of the SgrA West Mini-Spiral. Several helium stars have a radial velocity comparable to the velocity of the interstellar gas in which they are embedded. In the final discussion, all these findings are examined to present a possible scenario for the formation of very massive stars in the exceptional conditions of the vicinity of the central Black Hole.
\end{abstract}

Key words. instrumentation: spectrograph - techniques: radial velocities - infrared: stars - galaxy: center stars: early-type - stars: wolf-rayet

\section{Introduction}

The very inner region of the Galactic Center (GC) is the focus of many studies as it offers the unique opportunity to study star formation and the extreme gas kinematics peculiar to the vicinity of a $2.510^{6} M_{\odot}$ black hole (Genzel et al. 1997; Ghez et al. 1998). The presence of an unusually broad $2.058 \mu \mathrm{m}$ neutral helium line in emission was among the early known peculiarities of the central infrared source, originally called IRS 16 (Hall et al. 1982). Continuously improved spatial resolution has made it possible to tie this emission to individual stars and to suggest that this

\footnotetext{
Send offprint requests to: J. P. Maillard, e-mail: maillard@iap.fr

* Visiting Astronomer, Canada-France-Hawaii Telescope, operated by the National Research Council of Canada, le Centre National de la Recherche Scientifique de France and the University of Hawaii, USA.
}

emission is explained by the presence of massive, young, hot stars (Najarro et al. 1997a). However, even if the formation of high mass stars was favored in the GC (Morris 1993), the prediction of evolving starbursts cannot fully explain the large abundance of massive, emission-line stars which are normally very rare and short-lived (Lutz 1998). Therefore, more spectroscopic observations are warranted to better understand the unique conditions in the central parsec of the Milky Way which can lead to the formation of numerous helium emission-line stars. An exact census and a precise determination of the physical properties of these stars is also important since they should significantly contribute to the ionization of the central parsec.

In this paper, we present new data obtained with an original type of integral field spectrometer, an imaging Fourier Transform Spectrometer called BEAR, on the 3.6-m Canada-France-Hawaii Telescope. The use of this instrument represents an effort to make a significant step 
in associating the best possible spatial resolution and a high spectral resolution in the near infrared. The spatial resolution is not limited by a slit width, as with a standard grating spectrometer. It corresponds to the common seeing conditions at the $\mathrm{CFH}$ Telescope on Mauna Kea at $2 \mu \mathrm{m}\left(\simeq 0.6^{\prime \prime}\right)$. The spectral resolution is provided by the FTS. To complete the star detection, an adaptive optics (AO) image of the same field in the $K$ band was utilized. The processing of the BEAR data cube is described in Sect. 3. All the new results obtained from this study are presented in Sect. 4, including the display of the He I $2.058 \mu \mathrm{m}$ line profiles of all the detected stars and, for the first time, the mapping of flows of interstellar helium. A detailed review of the detected stars follows in Sect. 5 . Finally, a discussion of the nature of the He I stars, of the link between these stars and the helium flows, and of a possible star formation scenario are presented in Sect. 6 .

\section{Observations}

The 3-D data were obtained in July 25, 1997 during a run with the BEAR Imaging FTS at the $\mathrm{f} / 35$ infrared focus of the 3.6-m CFH Telescope. For a detailed description of the properties of this type of instrument, we refer the reader to Simons et al. (1994), Maillard (1995), and to an updated review in Maillard (2000). Briefly, the BEAR instrument results from the coupling of the CFHT step-scan high resolution facility FTS (Maillard \& Michel 1982) with a $256 \times 256 \mathrm{HgCdTe}$ facility camera. The field of view is circular with a $24^{\prime \prime}$ diameter, which corresponds to $0.93 \mathrm{pc}$ at an assumed distance of $8 \mathrm{kpc}$ for the GC (Reid 1993). The plate scale on the detector is $0.35^{\prime \prime} /$ pixel. The raw data consist of a cube of 300 planes with an integration time of $10 \mathrm{~s}$ per image, an image being taken at each stepping of the interferometer. In the camera, a narrowband filter (bandpass 4806-4906 $\mathrm{cm}^{-1}$ ) isolates the He I $2.058 \mu \mathrm{m}$ line. Observation of the GC from Mauna Kea is not possible at low airmass (at $42^{\circ}$ above horizon at its highest). Therefore, the scan was acquired with an airmass less than 1.8 to preserve the image quality. The maximum path difference which was reached corresponds to a limit of resolution $(F W H M)$ of $1.2 \mathrm{~cm}^{-1}$, i.e., $74 \mathrm{~km} \mathrm{~s}^{-1}$. Much higher spectral resolution can be obtained in this mode with the instrument (Maillard 2000). This value represents a compromise between the resolution needed to resolve the narrowest features of the line profiles and the detection depth. In any case, this resolution is at least 4 times better than in most of the previous spectral observations (Allen et al. 1990; Geballe et al. 1991; Krabbe et al. 1991; Krabbe et al. 1995; Blum et al. 1995b; Libonate et al. 1995; Tamblyn et al. 1996; Genzel et al. 1996; Najarro et al. 1997a, to quote the most important contributions to this study). A data cube on an A0 calibration star (HD 18881, $m_{K}=7.14$ from Elias et al. 1982) was obtained on the same night, at exactly the same spectral resolution. This procedure was important for the precise correction of telluric absorptions since the $2.058 \mu \mathrm{m}$ line is in the middle of a strong $\mathrm{CO}_{2}$ band.
High spatial resolution images of the inner region of the GC in the $K$ band were obtained with the CFHT Adaptive Optics Bonnette (Lai et al. 1997) equipped with the $1024 \times 1024 \mathrm{HgCdTe}$ KIR camera (Doyon et al. 1998) on 1998, 26 June. The total integration time is equal to $480 \mathrm{~s}$ from the acquisition of 4 times 10 exposures of $12 \mathrm{~s}$ each to cover a total field of $\simeq 40^{\prime \prime} \times 40^{\prime \prime}$, just a little bigger than the direct field of the camera $\left(35^{\prime \prime} \times 35^{\prime \prime}\right)$. The reference star for guiding was a $m_{K}=14.5$ star located $24^{\prime \prime}$ from SgrA*. The data processing included the filtering of star halos and the assemblage of the individual images to build the total field, which contains the entire BEAR field. The $F W H M$ of the point-spread function (psf) in the final image varies from $0.13^{\prime \prime}$ to $0.20^{\prime \prime}$, depending on the distance to the guiding star. A slight elongation can be seen on the most distant images. Nonetheless, the image quality is roughly 4 times better than the seeing-limited BEAR images.

\section{Processing of BEAR data}

The processing of the BEAR data included two major operations. The first part is the standard processing for any BEAR data cube, from the raw data which are interferograms to the spectral cube. A second part has had to be developed specifically to cancel the atmospheric $\mathrm{OH}$ emission, extract the stars from a very crowded field, and to separate, in the spectra, the stellar contribution from the background emission. All these steps are briefly described below.

\subsection{Cube reduction}

The cube reduction, made with a package called bearprocess (Maillard 2000), consists of the usual operations of flat-fielding of the images of the raw cube, sky subtraction, correction for bad pixels, and registration of each frame relative to the first one to correct for turbulent motions and flexure drifts. All the interferograms are extracted from this corrected cube, and the corresponding spectra are computed by FFT, leading in this case to a 384-plane cube, this number being determined as the sum of powers of two, just larger than the initial 300 planes. The same operations have been made on the reference star data cube to yield in the end a single spectrum. From the division of the GC spectral cube by this reference spectrum, a new cube was produced, corrected for the instrumental and atmospheric transmission. In order to fully reconstruct the line profiles, and to apply the instrumental phase correction through the field, an oversampled cube of 1153 frames was computed which contains only the useful part of the spectrum after division, between $4827.19 \mathrm{~cm}^{-1}$ $(2.0716 \mu \mathrm{m})$ and $4889.74 \mathrm{~cm}^{-1}(2.0451 \mu \mathrm{m})$. In this cube, the separation between frames corresponds to a mean velocity resolution of $3.35 \mathrm{kms}^{-1}$, i.e., an oversampling by a factor of 12 from the initial cube. 


\section{2. $\mathrm{OH}$ correction}

The CFHT-FTS is based on a design with dual input, dual output (Maillard \& Michel 1982). For observations of isolated objects the source is centered in one entrance aperture, while the other one is open on the sky $53^{\prime \prime}$ West. This makes an automatic correction of the sky emission possible, in particular for $\mathrm{OH}$. In the case of an extended field such as the GC, a single aperture must be open. Therefore, the $\mathrm{OH}$ emission strongly contaminates the raw data cube. The problem is particularly serious since a strong $\mathrm{OH}$ line falls at $2.0563 \mu \mathrm{m}$, within a typical linewidth of the stellar $\mathrm{He} \mathrm{I}$ line. This $\mathrm{OH}$ line is not resolved and thus appears as an extended sinc function, the natural instrumental lineshape of an FTS. In the useful part of the spectrum, a second $\mathrm{OH}$ line, four times fainter, at $2.0499 \mu \mathrm{m}$, falls in the continuum. In addition, the $\mathrm{OH}$ line intensities do not appear to be perfectly uniform over the entire field.

We applied a method intended to allow the best removal of these lines, secondary maxima included. First, the spectrum of the atmospheric emission $\left(S_{\text {sky }}\right)$ to be used as template was extracted by averaging the emission over about 100 pixels from small areas of the field devoid of sources. Then, for each pixel spectrum $S$ of the data cube, the following expression was generated, integrated in the wavenumber $\sigma$ over the full spectral range:

$E(\mu)=\int\left(\frac{\mathrm{d}^{2}\left(S-\mu S_{\text {sky }}\right)}{\mathrm{d} \sigma^{2}}\right)^{2} \mathrm{~d} \sigma$.

The final corrected spectrum is $S-\mu_{\mathrm{m}} S_{\text {sky }}$ where $\mu_{\mathrm{m}}$ corresponds to the value of $\mu$ for which $E(\mu)$ is minimum. A new cube cleaned of $\mathrm{OH}$ emission was created according to this procedure, to which all the subsequent operations were applied.

\subsection{Extraction of stellar spectra}

An image of the field of view was generated by co-adding most of the frames of this cube, with the exception of about 100 frames at each extremity, where division by the reference spectrum creates excessive noise. An automatic 2-D local maximum search was run on this image in order to detect the stars. With this procedure a total of 90 individual stars was identified within the circular field of the instrument. By using the photometry of Ott et al. (1999) for the faintest stars which are in common we determined a limiting magnitude of $m_{K} \simeq 13$ for the stellar flux integrated in a $3 \times 3$ pixels aperture (or $\left.\simeq 1^{\prime \prime} \times 1^{\prime \prime}\right)$. For almost the same field as us (a square field of $20^{\prime \prime} \times 20^{\prime \prime}$ centered on SgrA $\left.{ }^{\star}\right) 218$ stars brighter than $m_{K}=13$ are reported by Ott et al. (1999) from deconvolved images integrated over a $0.25^{\prime \prime}$ diameter aperture. Obviously, the main limitation comes from the seeing-limited imagery with BEAR in a very crowded field.

A facility program called cubeview (Maillard 2000), specially developed to inspect any BEAR data cube, was used to extract the 90 stellar spectra from the cube, by integration over a $3 \times 3$ pixels aperture, centered on the brightest pixel of each detected star image. The final spectra resulted from a smoothing operation (boxcar function) to improve the $S / N$ ratio. This operation was justified since the spectral resolution was much narrower than the broad stellar line profiles $\left(74 \mathrm{kms}^{-1}\right.$ against $\sim 1000 \mathrm{~km} \mathrm{~s}^{-1}$ ). To search all spectra for the presence of the He I $2.058 \mu \mathrm{m}$ line in emission, a $3 \sigma$-detection criterion was applied to each smoothed spectrum, with the noise estimated in the continuum. From the same cube a "line cube" was generated. This was done by estimating a linear continuum in each spectrum, extracted pixelby-pixel over the entire field, and by subtracting it from the original spectrum. Thus, the helium emission was all that remained. Using cubeview to inspect the cube images within the helium emission range revealed the stars being source of a He I emission as bright spots. Note that with spectro-imaging data the equivalent of an ideal square filter can be applied, isolating only the emission component without continuum, thus giving the maximum contrast to these stars, more accurately than could be done by imaging through a narrow-band filter. A few other stars with helium emission, for which the automatic detection had failed, were found by this method. Finally, the spectra of all the stars with helium emission were extracted from both cubes, in order to obtain two spectra for each star: the total spectrum, and the spectrum of the emission line only.

\subsection{Separation of stars and gas}

A co-added image was created with cubeview from all the frames of the line cube containing some He I emission. The resulting image clearly shows that the emission is concentrated in bright points, likely stars, but also in diffuse zones, indicative of interstellar gas lanes. Therefore, a separation of stars and gas must be conducted to obtain pure stellar line profiles and a spectral cube of the interstellar medium (ISM) emission only.

The He I line profiles detected with cubeview from the line cube in the gas patches exhibit a width just equal to the spectral resolution, which contrasts with the much broader profiles on most stellar points. The increase in spectral resolution provided by the BEAR spectrometer appears essential for distinguishing the ISM emission from the stellar emission. In many of the stellar profiles a narrow component is seen to be superimposed on a broad component. In these cases an inspection of the data in the vicinity of the star confirms the presence of extended ISM emission along the line of sight. Hence, the stellar profile can be cleaned of the ISM emission contribution by a local interpolation on the profile. In other cases, a line with width equal to the spectral resolution appears on top of a stellar continuum. This typical linewidth avoids confusion with an emission of stellar origin. However, in a few cases the emission line on top of a stellar continuum appears relatively narrow, about twice as wide as a typical ISM line. Only the absence of ISM emission in the 
neighborhood of such a star gives confidence in the stellar nature of the emission. The ISM emission can also mimic a stellar profile. Indeed, inspection of the cube indicates that, in some locations, the ISM emission shows several velocity components, which can merge into a broader line. In these cases a global inspection of the images confirms that the profile is due to ISM emission only. Finally, after all this careful selection, a fit to the stellar emission at all the confirmed He I star positions in the line cube was subtracted from the spectra, generating a spectral cube of the ISM emission.

\subsection{Fitting of the $2.058 \mu \mathrm{m}$ emission line profiles}

In the end, 16 stars from the 90 stars isolated in the $\sim 1$-pc field centered on $\mathrm{SgrA}^{\star}$ exhibit the $2.058 \mu \mathrm{m}$ He I line in emission, which can be attributed only to the underlying star. A homogeneous set of fully resolved line profiles is obtained. This signature justifies them being called "helium stars". Actually, this designation is not a true stellar classification. They are simply stars showing the $2.058 \mu \mathrm{m}$ helium line in emission. In the following the term "helium star" or "He I star" will therefore be adopted in this context. From the stellar positions, the correlation with previously identified stars was made from photometric surveys, e.g., Blum et al. (1996a), Eckart \& Genzel (1997), and Ott et al. (1999). Three new helium stars not present in prior lists were first noted: HeI N1, HeI N2 and HeI N3. One of them, HeI N1, coincides with ID 180, a source already identified in the photometric survey of Ott et al. (1999). This identification was adopted.

In order to derive the radial velocities of all these stars, simple analytical models were used which take into account the $\mathrm{P}$ Cygni profile evident in most profiles. The $2.058 \mu \mathrm{m}$ He I line has the advantage of not being blended with the emission lines of other atomic species which are likely to be present in the spectrum of these stars (Najarro et al. 1997a). Depending on the profile shape, we used three types of fitting models (a, b, c). In each case, the model yields the $F W H M$ of the emission component, and the velocity domain of the full profile, FWZI (full width at zero intensity). The center of FWZI defines the radial velocity $\left(V_{\mathrm{R}}\right)$ of the star. FWZI is also indicative of the terminal outflow velocity of the expanding envelope.

a) For the few profiles with no detectable absorption component, a fitting by a Gaussian profile was used. In this case the FWZI is in fact estimated at one hundredth of the maximum intensity above the baseline;

b) For the P Cygni profiles for which the absorption component has a width comparable to that of the emission component, a two-Gaussian fitting was applied. FWZI is limited with the same criterion as above on the emission side, and at one hundredth of the central depth below the baseline on the absorption side;

c) For the stars with a $\mathrm{P}$ Cygni profile but showing a very broad emission component, a two-component fitting was used with a profile described in Morris (1985) for the emission component, and a Gaussian profile for the absorption component. For the determination of FWZI, the boundary of the profile on the red side is given by the emission fit. The Gaussian profile is limited as above on the absorption side.

\subsection{Calibration of line profiles}

For flux calibration, the stellar spectra must be extracted from the cube which includes the continuum. However, the intensity in these spectra is contaminated by the nearby stars present in the $3 \times 3$ pixel BEAR box centered on each helium star. Corrections of this contamination were applied with the help of the AO image. In order to do this, the star positions and the peak intensities in the AO image were all determined with an automatic procedure. Then, at each star position, the BEAR psf was placed, which is a Lorentzian profile obtained from the calibration star data cube with the corresponding intensity. The result is an image at the BEAR spatial resolution. This reconstructed image was superimposed upon the image obtained by co-adding the frames of the BEAR cube with only the stellar continuum, by looking for the best match of the star patterns between the two images. By this process we determined the appropriate offsets and rotation between the two images. With these registration parameters, the BEAR box for each He I star, centered on the bright pixel determined from the line cube was projected on the corresponding area of the original AO image. From this superposition, in the case of multiple images, the most likely identification of the helium star can be asserted, and then the contamination by the neighboring stars within the square aperture estimated. The absolute star positions could be measured at the precision of the pixel size in the AO image $\left(0.035^{\prime \prime}\right.$ pixel). They were determined first as offsets with respect to IRS $16 \mathrm{NE}$, because this star from the AO image is a relatively isolated, bright star. Its position was taken from Ott et al. (1999), but in that paper, all the positions are given as offsets relative to the brightest local source, IRS 7, which is unusable in the AO image because its image is saturated. In order to present the final offsets of all the sources with respect to $\operatorname{Sgr} A^{\star}$, the position of IRS 7 relative to $\mathrm{SgrA}^{\star}$ was taken from Menten et al. (1997) and used to derive the IRS 16NE position relative to $\operatorname{Sgr}^{\star}$.

\section{Presentation of results}

\subsection{Two classes of Hel stars}

By inspecting the shapes of the full set of He I line profiles, two classes of stars can be clearly distinguished. Indeed, 7 stars show a narrow emission line with a mean $F W H M$ of $225 \pm 75 \mathrm{kms}^{-1}$, and 9 stars a very broad emission line with a mean $F W H M$ of $1025 \mathrm{~km} \mathrm{~s}^{-1}$, all the values being within $\pm 400 \mathrm{~km} \mathrm{~s}^{-1}$ of this average. The FWZI associated with the latter group varies from $\sim 1700 \mathrm{~km} \mathrm{~s}^{-1}$ to $\sim 3000 \mathrm{~km} \mathrm{~s}^{-1}$. The stars of each group are gathered 


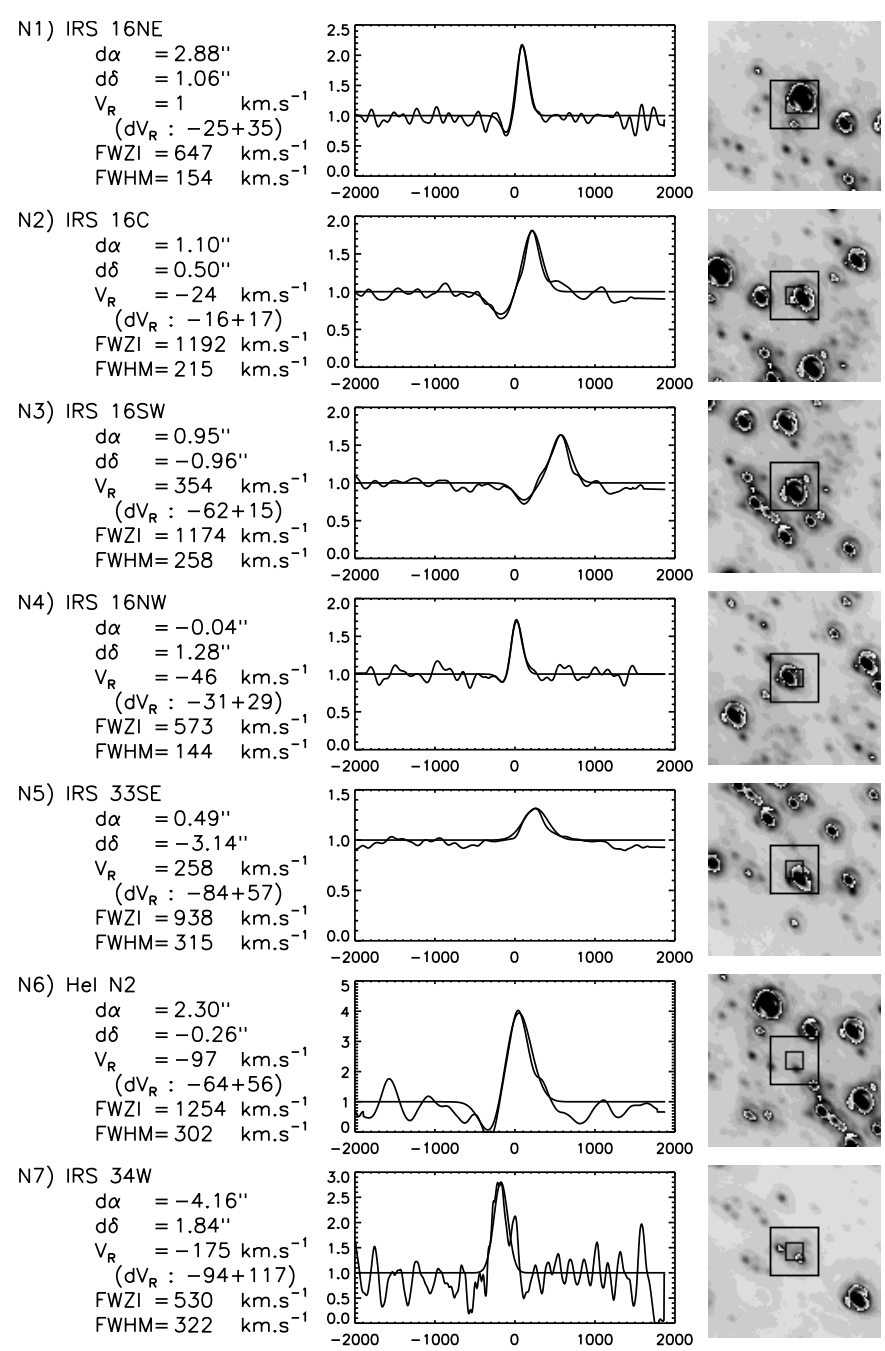

Fig. 1. Identification of HeI stars with a narrow-line profile and adaptive optics image of the area. The star positions $(\mathrm{d} \alpha$, $\mathrm{d} \delta$ ) are offset with respect to $\mathrm{SgrA}^{\star}$ estimated from their counterpart in the AO image (see Sect. 3.6). The small box in each image shows the size of one BEAR pixel, corresponding to the brightest pixel detected at the star position in the "line cube" (see Sect. 3.3). The $3 \times 3$ pixel square represents the box in which the spectrum has been extracted. An analytical profile is fitted to each profile with parameters described in Sect. 3.5, given in the left column. $V_{\mathrm{R}}$ is the radial velocity with $\mathrm{d} V_{\mathrm{R}}$ the 1- $\sigma$ error bar (see Sect. 4.3). The vertical scale in each spectrum is given in multiples of the intensity of the neighboring continuum, for which the calibrated value is given in Table 1

in Figs. 1 and 2 respectively, with the parameters of the fitting of the line profiles, the star names, and the star positions as offsets from $\operatorname{Sgr} A^{\star}$. These positions are given in arcsecs at the precision of the position of their counterpart measured in the AO image (Sect. 3.6).

Note that most of these profiles are of the common P Cygni variety, with the standard absorption on the blue side. This absorption is generally shallow for the very broad emission lines, since the emission almost fills the absorption width, and is deeper for the narrow emission lines. For the broad-line profiles (Fig. 2) a flat top is seen
B1) ID 180
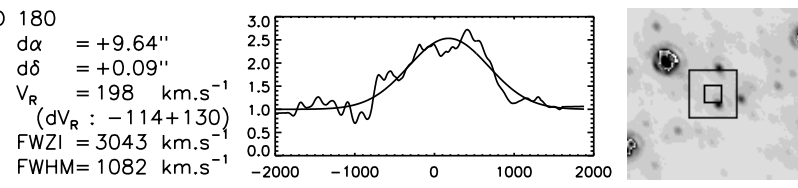

B2) IRS 7E2
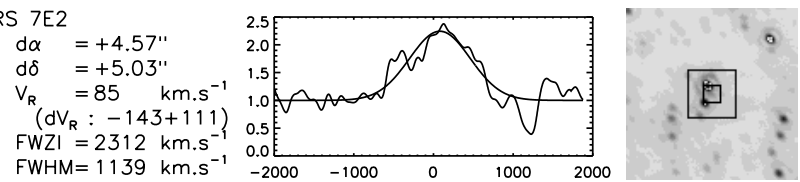

B3) IRS $9 W$

$$
\begin{aligned}
& \mathrm{d} \alpha=+2.67^{\prime \prime} \\
& \mathrm{d} \delta \quad=-5.80^{\prime \prime} \\
& \mathrm{V}_{\mathrm{R}}=221 \mathrm{~km} \cdot \mathrm{s}^{-1} \\
& \left(\mathrm{dV}_{\mathrm{R}}:-53+57\right) \\
& \mathrm{FWZI}=2010 \mathrm{~km} \cdot \mathrm{s}^{-1} \\
& \mathrm{FWHM}=1408 \mathrm{~km} \cdot \mathrm{s}^{-1}
\end{aligned}
$$

B4) IRS $15 \mathrm{SW}$

$$
\begin{aligned}
\mathrm{d} \alpha & =-1.38^{\prime \prime} \\
\mathrm{d} \delta & =+10.36^{\prime \prime} \\
\mathrm{V}_{\mathrm{R}} & =-179 \mathrm{~km} \cdot \mathrm{s}^{-1} \\
\left(\mathrm{dV} \mathrm{V}_{\mathrm{R}}:-26+24\right) & -26+2166 \mathrm{~km} \cdot \mathrm{s}^{-1} \\
\mathrm{FWZI} & =2166 \mathrm{~km} \cdot \mathrm{s}^{-1} \\
\mathrm{FWHM} & =894
\end{aligned}
$$
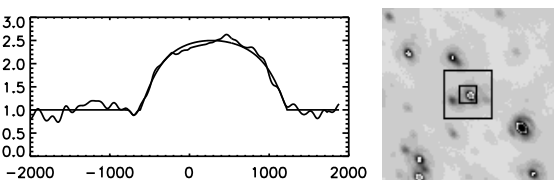

B5) IRS $13 E 3$

$$
\begin{aligned}
& \mathrm{d} \alpha=-3.37^{\prime \prime} \\
& \mathrm{d} \delta=-1.39 " \\
& \mathrm{~V}_{\mathrm{R}}=27 \mathrm{~km} \cdot \mathrm{s}^{-1} \\
& \left(\mathrm{dV}_{\mathrm{R}}:-72+48\right) \\
& \mathrm{FWZI}=1752 \mathrm{~km} \cdot \mathrm{s}^{-1} \\
& \mathrm{FWHM}=974 \mathrm{~km} \cdot \mathrm{s}^{-1}
\end{aligned}
$$

B6) IRS $7 W$

$$
\begin{aligned}
& \mathrm{d} \alpha=-3.99^{\prime \prime} \\
& \mathrm{d} \delta=+5.25^{\prime \prime} \\
& \mathrm{V}_{\mathrm{R}}=-292 \mathrm{~km} \cdot \mathrm{s}^{-1} \\
& \left(\mathrm{dV}_{\mathrm{R}}=-41+49\right) \\
& \mathrm{FWZI}=1868 \mathrm{~km} \cdot \mathrm{s}^{-1} \\
& \mathrm{FWHM}=1031 \mathrm{~km} \cdot \mathrm{s}^{-1}
\end{aligned}
$$

B7) AF

$$
\begin{aligned}
& \mathrm{d} \alpha=-6.94^{\prime \prime} \\
& \mathrm{d} \delta=-6.82^{\prime \prime} \\
& \mathrm{V}_{\mathrm{R}}=192 \mathrm{~km} \cdot \mathrm{s}^{-1}=192 \\
&\left(\mathrm{dV}_{\mathrm{R}}:-64+52\right) \\
& \mathrm{FWZI}=1931 \mathrm{~km} \cdot \mathrm{s}^{-1} \\
& \mathrm{FWHM}=826 \mathrm{~km} \cdot \mathrm{s}^{-1}
\end{aligned}
$$
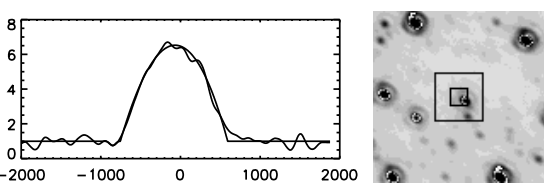

B8) AF NW

$\mathrm{d} \alpha=-7.97^{\prime \prime}$

d $\delta=-3.35^{\prime \prime}$

$V_{R}=159 \mathrm{~km} \cdot \mathrm{s}^{-1}$ $\left(d V_{R}:-79+64\right)$ $F W Z I=1871 \mathrm{~km} \cdot \mathrm{s}^{-1}$ $F W H M=1300 \mathrm{~km} . \mathrm{s}^{-1}$
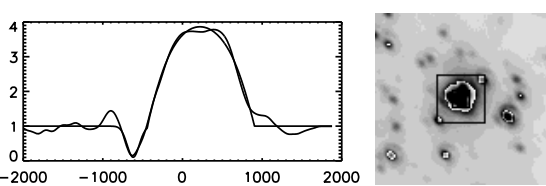

B9) $\mathrm{Hel} \mathrm{N3}$
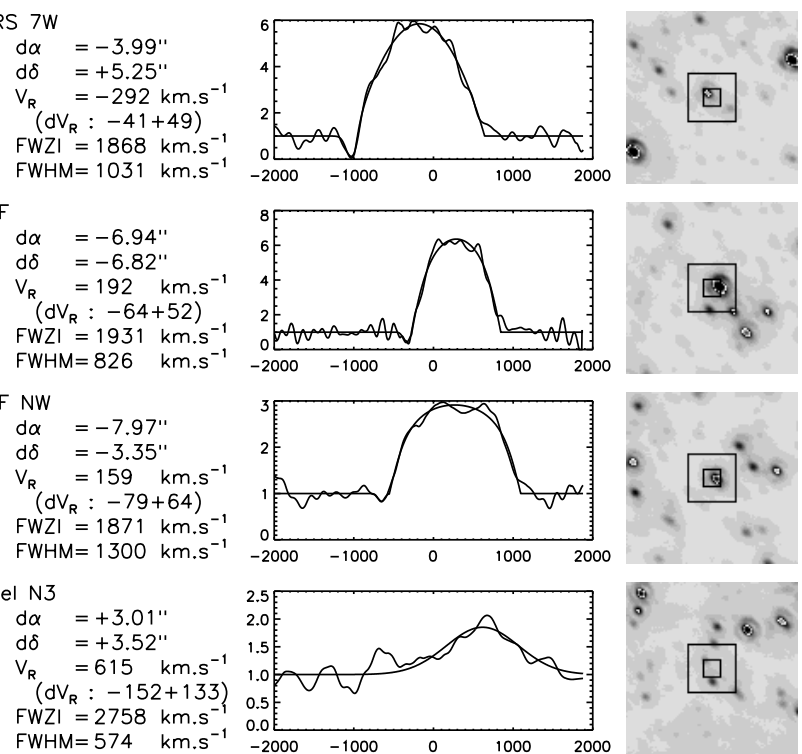

Fig. 2. Identification of He I stars with a broad-line profile and adaptive optics image of the area. The projected boxes have the same meaning as in Fig. 1

for AF, which was already known (Najarro et al. 1994), but also for AF NW, IRS 7W, IRS 13E and presumably ID 180. All these various types of profile are encountered in models of P Cygni profiles (Castor \& Lamers 1979).

Table 1 presents the continuum flux level for each star measured at $2.06 \mu \mathrm{m}$, at wavelengths just outside of the emission profile, estimated by the procedure described in Sect. 3.6. No extinction correction has been applied. As these stars are located at the same distance, a comparison of flux is possible without correction. From an examination of Table 1, it appears that with this distinction of 
Table 1. Continuum $\Phi$ of the He I stars at $2.06 \mu \mathrm{m}$

\begin{tabular}{|lll|lll|}
\hline \multicolumn{3}{|c|}{${\text { Narrow-line } \text { stars }^{a}}^{\text {ID }}$} & Name & \multicolumn{3}{c|}{$\Phi^{c}$} & ID & Name & $\Phi^{c}$ \\
\hline \hline N1 & IRS 16NE & 25.93 & B1 & ID 180 & 0.59 \\
N2 & IRS 16C & 13.23 & B2 & IRS 7E2 & 0.77 \\
N3 & IRS 16SW & 10.87 & B3 & IRS 9W & 1.55 \\
N4 & IRS 16NW & 9.41 & B4 & IRS 15SW & 1.02 \\
N5 & IRS 33SE & 8.52 & B5 & IRS 13E3 & 2.26 \\
& & & B6 & IRS 7W & 0.98 \\
N6 & HeI N2 & $(0.76)$ & B7 & AF & 3.88 \\
N7 & IRS 34W & $(1.76)$ & B8 & AF NW & 1.84 \\
& & & B9 & HeI N3 & 0.58 \\
\hline \multicolumn{3}{|c|}{ mean $^{*}$} & 13.59 & \multicolumn{3}{c}{ mean } & 1.50 \\
\hline
\end{tabular}

${ }^{a}$ See Fig. 1.

${ }^{b}$ See Fig. 2 .

${ }^{c} 10^{-14} \mathrm{~W} \mathrm{~m}^{-2} \mu \mathrm{m}^{-1}$.

* Mean intensity estimated without N6 and N7 (see text).

two families of line profile is associated another clear difference which had not been noticed before, namely the level of continuum. The continuum of the stars having a narrow profile is bright and with a comparable intensity, except for IRS 34W and HeI N2, which have a definitely weaker continuum. The continuum of the broad-line stars is fainter by a factor 9.0 on average $(\simeq 2.4 \mathrm{mag})$ than that of the narrow-profile stars. The AF star and IRS 13E3 appear to be the brightest objects of this group, though their continuum intensity is weaker by more than a factor 3 than the mean value of the narrow-line category. We return to these particular cases below.

The $K$-band AO image gives the opportunity of estimating the $K$ magnitude, without extinction correction, of the HeI stars. The photometric calibration was made by looking in the Ott et al. (1999) survey for a bright star common to our list, which is sufficiently isolated, and for which the photometry indicates a low index of variability. IRS $16 \mathrm{NE}$ was chosen as reference star, from which the $K$ magnitude of all the other stars was deduced. With the same presentation as Table 1 , the results are reported in Table 2 . The mean difference of $K$ magnitude between the two classes is equal to 2.18 . That corresponds to a ratio of 7.45 against 9.0 measured near $2 \mu \mathrm{m}$. This difference is due to the fact that, in the flux reported in Table 1, the correction of the contribution of neighboring stars (Sect. 3.6) can be made only by assuming the same spectral distribution in the $K$ band for these stars and the He I star, which is an approximation. For example, IRS 13E3, which was the second brightest star among its group from Table 1, is not so prominent in $K$. Only AF remains 1 mag above the average value. However, the general trend observed at $2 \mu \mathrm{m}$ is largely confirmed. Note that IRS $16 \mathrm{SW}$ is found 0.2 magnitude brighter than the mean value reported by Ott et al. (1999), which is well within the range of
Table 2. $K$ magnitude of the He I stars

\begin{tabular}{|lll|lll|}
\hline \multicolumn{3}{|c|}{ Narrow-line stars } & \multicolumn{3}{c|}{ Broad-line stars } \\
\hline ID & Name & $m_{K}$ & ID & Name & $m_{K}$ \\
\hline \hline N1 & IRS 16NE & $8.76^{a}$ & B1 & ID 180 & 12.12 \\
N2 & IRS 16C & 9.41 & B2 & IRS 7E2 & 11.93 \\
N3 & IRS 16SW & 9.38 & B3 & IRS 9W & 11.62 \\
N4 & IRS 16NW & 9.80 & B4 & IRS 15SW & 11.21 \\
N5 & IRS 33SE & 9.75 & B5 & IRS 13E3 & 11.73 \\
& & & B6 & IRS 7W & 11.85 \\
N6 & HeI N2 & $(12.47)$ & B7 & AF & 10.56 \\
N7 & IRS 34W & $(11.56)$ & B8 & AF NW & 11.52 \\
& & & B9 & HeI N3 & 12.47 \\
\hline \multicolumn{3}{|c|}{ mean $^{*}$} & 9.35 & \multicolumn{4}{|c}{ mean } & 11.53 \\
\hline
\end{tabular}

${ }^{a}$ From Ott et al. (1999).

* Mean magnitude estimated without N6 and N7 (see text).

periodic variation reported for this star. The source ID 180 is found to be $\sim 0.6$ mag brighter than in Ott et al. (1999), while AF has exactly the same magnitude.

\subsection{Comparison with previous lists of Hel stars}

The recent papers dedicated to surveying the helium stars in the central region of the GC are those of Krabbe et al. (1995), Tamblyn et al. (1996), Blum et al. (1996a) and Eckart \& Genzel (1997). Blum et al. (1996a) present the most complete compilation of identified stars with their spectral type, reflecting both their own work and that of others. In this list, for the first time, the helium stars are identified under two denominations: He I and WC9. The latter is a sub-type of Wolf-Rayet stars (WR) which in addition to the He I $2.058 \mu \mathrm{m}$ line in emission have the CIII and the CIV lines in their $K$-band spectrum. A comparison of the list of stars that we identify as genuine He I stars with the list of Blum et al. (1996a) shows that several of their stars are missing. A few candidates were just at the edge of our field and cannot be confirmed. But it turns out that, in the other cases, the detected emission line can be interpreted as due to the ISM emission and not to the star, or to the background contamination from a nearby He I star. Following primarily the list of helium stars reported in Blum et al. (1996a), either as He I or as WC9 stars, then in Eckart \& Genzel (1997), and at last in Tamblyn et al. (1996), all the stars absent from our list are worth a special comment:

- IRS 1W: We confirm that IRS 1W, which is in the list of Krabbe et al. (1995) but not in Blum et al. (1996a), where it is given as a red star, is indeed not a He I star. Blum et al. (1995b) had already shown a spectrum of IRS $1 \mathrm{~W}$ with no intrinsic He I $2.058 \mu \mathrm{m}$ emission line and Libonate et al. (1995) had also cast doubt on the notion that IRS $1 \mathrm{~W}$ was a compact He I emissionline star. That is a case where the line present in the raw spectrum at the star position is very narrow. The study of the vicinity clearly shows that this emission belongs to an ISM gas lane. From the slope of the 
continuum spectrum, polarization measurements, and a broadened image profile at high spatial resolution, Ott et al. (1999) suggest that IRS $1 \mathrm{~W}$ is embedded in a hot dust shell. However, a maximum of the ISM emission coincides with its position;

- BSD WC9: Blum et al. (1995a) have presented the $K$-band spectrum of this source as an example of a genuine WC9-type star. The same source is named Blum-WC9 by Tamblyn et al. (1996). A nearby source $\left(0.6^{\prime \prime} \mathrm{W}\right.$ and $\left.0.4^{\prime \prime} \mathrm{S}\right)$ is listed as BSD WC9B, which is supposed to be of same type. These two sources fall at the edge of our field, and therefore cannot be considered in our list;

- IRS 6E: This source is also reported as a WC9 star. There is a rather broad $\left(\simeq 270 \mathrm{~km} \mathrm{~s}^{-1}\right)$ emission feature but with two maxima in the spectrum toward IRS $6 \mathrm{E}$. The helium streamers are complex in its vicinity. The star lies just between the Bar and the mini-cavity. We interpret these two peaks as two velocity components of the ISM emission;

- IRS 29N: We do not confirm a helium star at the IRS 29N position, which is listed as a WC9 star by Blum et al. and in Eckart \& Genzel (1997). Separated by $0.5^{\prime \prime}$, IRS $29 \mathrm{~S}$ is identified as an MIII star by Krabbe et al. (1995). There is no ISM helium emission there. This source is located in the neighborhood of the bright helium stars in the IRS 16 cluster, so the reported detection can probably be explained as contamination in the Krabbe et al. data by the nearby helium stars;

- MPE-1.0-3.5: This source is listed as a WC9 star. In Ott et al. (1999) a star (ID $77, m_{K}=11.6$ ) coincides within $0.2^{\prime \prime}$, so it is probably the same source. In our data this star does not show any He I $2.058 \mu \mathrm{m}$ emission feature. Since it is close to the bright source IRS $16 \mathrm{NW}$, it was reported as a helium star probably for the same reason as the previous source;

- IRS 15NE: This star falls at the edge of the field and cannot be included in our list;

- MPE+1.6-6.8: A local maximum of the ISM emission is seen at the star position, but no stellar emission, which would reveal itself by a much larger width. This relatively bright star in $K$ (10.56 from Ott et al. 1999) might be another embedded star like IRS $1 \mathrm{~W}$;

- IRS 16CC: No He I stellar profile is found exactly at the position of this star;

- OSU He1: The helium line observed on the line of sight to this star is narrow and comes from the He I Mini-Spiral;

- IRS 16SE: This source appears in a star list restricted to the very inner region studied by Eckart \& Genzel (1997), who mention 3 He I stars, IRS 16SE1, IRS 16SE2 and a nameless source located 0.91" East and $1.99^{\prime \prime}$ North of SgrA*. All these sources lie in the proximity of strong HeI stars and in the Mini-Spiral. We do not confirm them as He I stars.

Tamblyn et al. (1996) used an attractive method to find HeI star candidates by associating an image taken through a narrow-band filter centered on the $2.058 \mu \mathrm{m}$ line. However, their contrast was not sufficient to detect the ISM emission and they wrongly claimed that "the majority of the HeI emission is from point sources". In their list, they report 5 supposed new identifications they named GCHe1 to GCHe5. By inspecting the 5 positions we find that GCHe2 (or TAM HeI in Blum et al. 1996a) is in fact IRS $9 \mathrm{~W}$, which is confirmed as a He I star, GCHe3 is IRS 33SE and GCHe4 likely AF NW. Regarding GCHe1, Tamblyn et al. (1996) indicate a position not more precisely than $1.1^{\prime \prime} \mathrm{NE}$ of AF. This region is at the very edge of our field. The current data do not allow us to confirm the presence of a helium star there. GCHe5 is identical in position to MPE-1.0-3.5, which has been rejected.

In conclusion, 6 out of 20 early-type stars listed in Blum et al. (1996a) as helium stars (noted He I or WC9) are not reported in our list. Three stars were excluded from our compilation, because they lie at the edge of the clear field: BSD WC9, BSD WC9B and IRS 15NE. Thus, 11 stars are in common. Of the 21 helium stars of Krabbe et al. (1995) we retain 13 sources. With 3 new stars which are added, the total number of helium stars in the central cluster remains roughly unchanged, but certainly not increased. However, this revision can modify some of the conclusions on this peculiar population.

\subsection{Examination of the stellar radial velocities}

With the radial velocities of all the helium stars reported by Eckart \& Genzel (1997), considered as the most recent estimations, and in Krabbe et al. (1995) when the value was missing from Eckart \& Genzel (1997), we have constructed a plot to compare to our estimations (Fig. 3). The error bars of each measurement are given, which put each star symbol at the center of an error box. In our work the error bars of $V_{\mathrm{R}}$ reported in Figs. 1 and 2 were estimated by shifting each best-fit profile in velocity, so that the maximum error in the obs.-calc. curve was within $\pm 1 \sigma$ of the noise. We can notice that our error bars are generally smaller than those reported earlier. However, the diagonal line does not cross the error box for 3 stars: N2, N5 and B2, i.e., IRS 16C, IRS 33SE and IRS 7E2. For either N2, which has a particularly narrow error bar because the emission is strong, or N5, the stellar profile has been corrected for the ISM emission. That may explain the observed discrepancy if in Eckart \& Genzel's work this correction was not done. We return to the case of N5 in Sect. 5.1. No correction for ISM emission has had to be made for B2. The low contrast of the emission line explains a wider error bar. However, the difference of reported $V_{R}$ has no obvious explanation, unless IRS 7E2 is another spectroscopic binary, which gave two different radial velocities when observed at two different epochs (difference of $\simeq 200 \mathrm{~km} \mathrm{~s}^{-1}$ ). Further observations are needed to assess this plausible hypothesis. 


\subsection{Location and kinematics of the Hel stars}

From the offsets given in Figs. 1 and 2, a map of the $\mathrm{He}$ I stars centered on $\mathrm{SgrA}^{\star}$ is presented in Fig. 4. The two classes of stars are distinguished by different symbols. Another property becomes apparent on this map. The narrow-line stars are grouped into a central compact cluster, in the IRS 16 region. Actually, 4 of them are designated as being components of IRS 16 . The new star HeI N2 is in the middle of them. The most external sources are IRS 33SE and IRS 34W, located just a few arcseconds South and West, respectively of the IRS 16 cluster. On the contrary, the broad-line stars are randomly distributed at the periphery of the field, beyond an inner radius of $\simeq 0.3 \mathrm{pc}$ from $\mathrm{SgrA}^{\star}$. Thus, the two or three emission-line stars missing because located at the edge of the observed field (Sect. 4.2) should also belong to the broad-line group.

In Fig. 5 are placed all the radial velocities $\left(V_{\mathrm{R}}\right)$ reported in Figs. 1 and 2, with their error bars, as a function of the dec-offset of the sources from $\operatorname{SgrA}^{\star}$. This plot is constructed with the same axes as a comparable diagram in Genzel et al. (1996) for the early-type stars. According to these authors all the stars with a positive velocity are concentrated in the upper left quadrant while the stars with a negative velocity are in the lower right quadrant. They conclude that this diagram shows the signature of a coherent retrograde motion of all early-type stars - a population of stars which contains mostly the He I stars - around an approximately East-West axis of rotation through $\mathrm{SgrA}^{\star}$. From our equivalent diagram (Fig. 5) with the velocities of the 16 confirmed He I stars, we note that stars are present in all 4 quadrants, with, however, a trend to be mostly distributed along a diagonal through the opposite upper left and lower right quadrants, which is consistent with a revised version of the same diagram by Genzel et al. (2000).

\subsection{Absorption line stars}

From the systematic inspection of the 90 stellar spectra extracted from the data cube, it appears that some stars might present a broad absorption feature at the position of the He I $2.058 \mu \mathrm{m}$ line. Tamblyn et al. (1996), and more completely Hanson et al. (1996) have observed all types of OB stars in the $K$ band. They show that giant and supergiant O-type stars, roughly from $\mathrm{O} 5$ to $\mathrm{O} 9$ may have the HeI $2.058 \mu \mathrm{m}$ line in weak absorption. The search for such stars was conducted with the same method as the one applied to the emission line stars. Similarly, a smoothing of the raw spectra was made since the absorptions are broad (FWZI between $\sim 900$ and $1500 \mathrm{~km} \mathrm{~s}^{-1}$ ) and shallow. The more noticeable detections correspond to the sources: IRS 7SE, IRS 14SW, MPE-1.1-2.2 and IRS 3. From the photometric survey of Ott et al. (1999), these stars are relatively bright $\left(m_{K}\right.$ between 10 and 11.3) with a low index of variability. Note that IRS $14 \mathrm{SW}$ and IRS 3 are indicated as cool stars in Blum et al. (1996a),

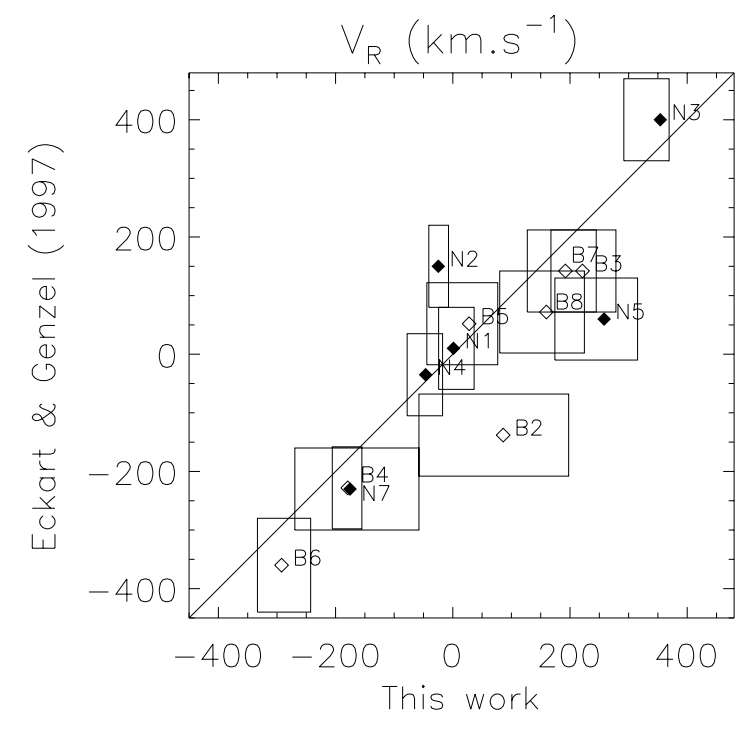

Fig. 3. Comparison of the estimated radial velocities $V_{\mathrm{R}}$ with their error boxes for the stars in common between our work and Eckart \& Genzel (1997). When not available in that paper, the values are from Krabbe et al. (1995). The narrow-line stars are represented by a filled diamond, the broad-line stars by an open diamond. See Figs. 1 and 2 for the star identifications

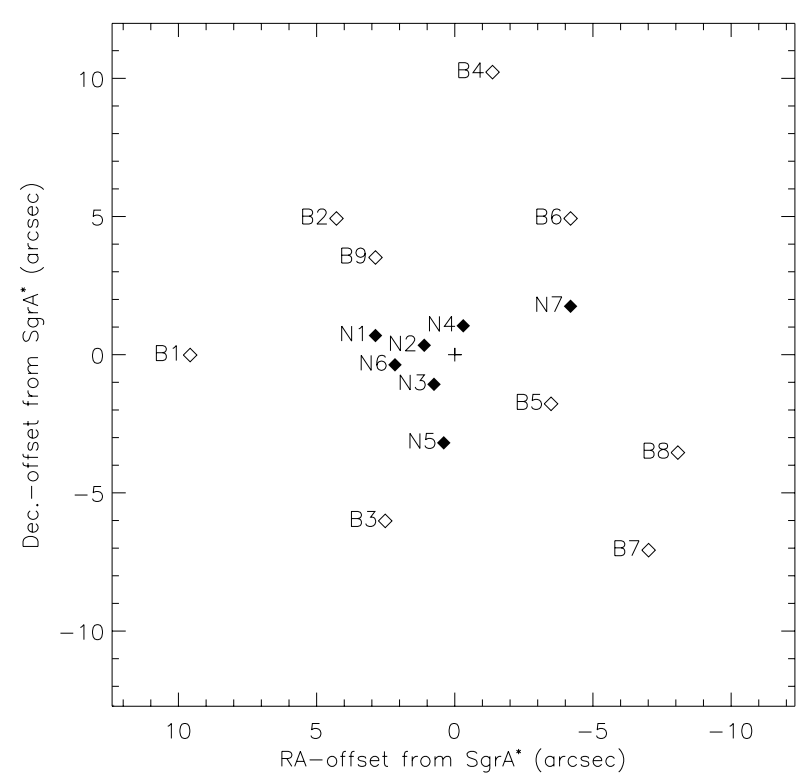

Fig. 4. Spatial distribution of helium stars from the offsets reported in Figs. 1 and 2, with respect to $\mathrm{SgrA}^{\star}$ marked by a cross. The symbols have the same meaning as in Fig. 3

with the latter characterized as "embedded" by Eckart \& Genzel (1997). Hence, this absorption can be of an origin other than photospheric helium. However, IRS 7SE and MPE-1.1-2.2 remain as potential OB star candidates. Observations over a wider spectral range are necessary to confirm the spectral nature of these two sources, which deserve further attention as possible indicators of the presence of O-type stars in the central cluster. 


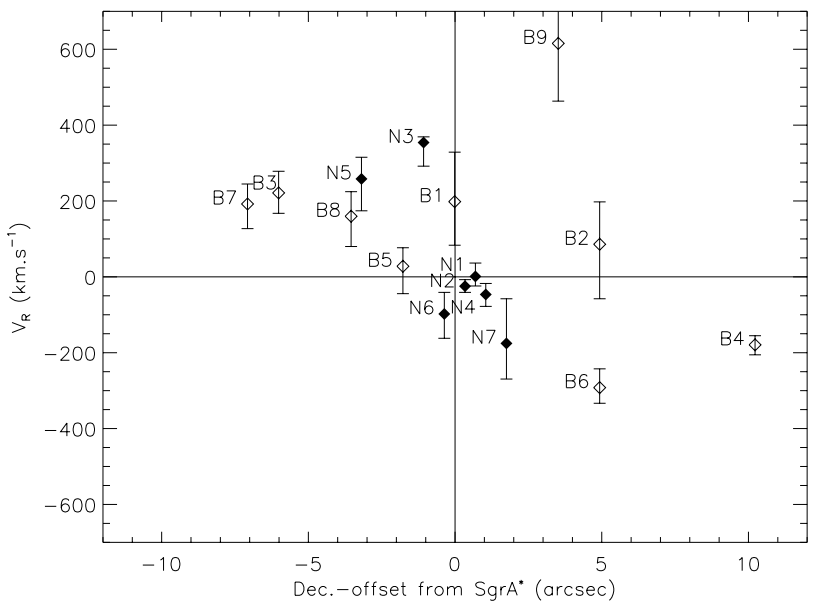

Fig. 5. Radial velocities with their error bar of the helium stars as a function of the dec.-offset from SgrA* (see Figs. 1 and 2). Filled and open diamonds have the same meaning as in Figs. 3 and 4

\subsection{Helium streamers}

An image of the interstellar helium emission is shown in Fig. 6. It was constructed by putting at each pixel the peak value of the corresponding spectrum in the ISM cube. The emission appears clearly distributed in coherent gas lanes. By comparing with the interstellar emission previously mapped in $\mathrm{Ne}^{+}$at $12.8 \mu \mathrm{m}$ by Lacy et al. (1991) and in $\operatorname{Br} \gamma$ (Morris \& Maillard 2000 and references therein) we see that this emission emanates from the Mini-Spiral, particularly prominent the Northern arm and the Bar. The mini-cavity can be also recognized. The Eastern arm is weak, except in an elongated feature at its western tip, at $\mathrm{d} \alpha=-1.5^{\prime \prime}, \mathrm{d} \delta=-2.5^{\prime \prime}$, showing a very distinct and extreme redshifted velocity. This component appears clearly in the integrated ISM velocity profile shown in Fig. 7, where it forms a separated maximum at $+275 \mathrm{~km} \mathrm{~s}^{-1}$. The same peak of velocity is observed in the $\operatorname{Br} \gamma$ data (Morris \& Maillard 2000) at the same position on the hydrogen streamers. The full range of velocity covered by the HeI mini-spiral is identical to the range measured in $\operatorname{Br} \gamma$, i.e., $-400,+400 \mathrm{~km} \mathrm{~s}^{-1}$ (Morris \& Maillard 2000). However, the helium ISM emission appears simpler than the $\operatorname{Br} \gamma$ emission, as attested by the line profile extracted at each pixel which appears always as a single and narrow emission peak, except where the main streamers are crossing. For example, three components are exceptionally observed where the Northern arm, the Bar and the feature of the Eastern arm are superimposed, at $\mathrm{d} \alpha=3^{\prime \prime}$, $\mathrm{d} \delta=-6^{\prime \prime}$. We already mentioned that the linewidth of a single component is exactly equal to the current limit of resolution. We can conclude that the velocity width of the helium streamers is certainly $<70 \mathrm{~km} \mathrm{~s}^{-1}$, however without being much narrower, since no sinc function profile is observed.

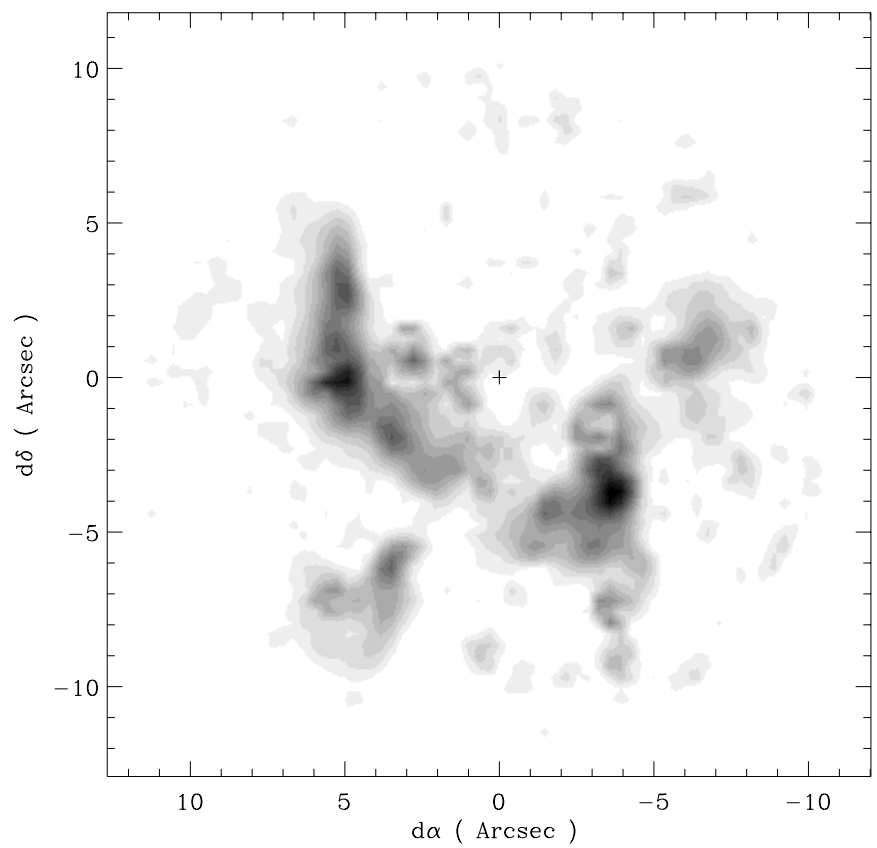

Fig. 6. Image of the helium streamers in the $2.058 \mu \mathrm{m}$ line. The emissions of stellar origin have been subtracted as much as possible. Small residuals remain. The position of $\mathrm{SgrA}^{\star}$ is marked by a cross at the center of the field

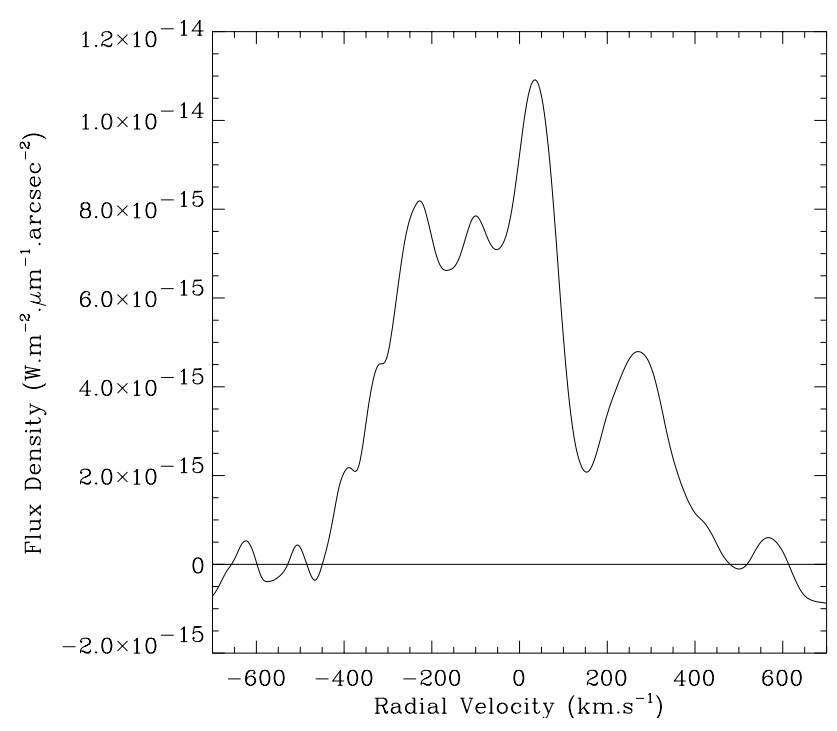

Fig. 7. Full velocity profile of the HeI Mini-Spiral obtained by extracting the spectrum from the ISM cube over a mask covering most of the emission. The calibrated flux density is an average by $\operatorname{arcsec}^{2}$ over the area of this mask

\subsection{Comparison of the radial velocities of the $\mathrm{Hel}$ stars and the local ISM}

As already mentioned, for several fully resolved He I line profiles, a narrower emission line from a helium streamer is seen superimposed on the stellar profile. By comparing the $V_{\mathrm{R}}$ of these stars derived from their $\mathrm{P}$ Cygni profile emission (Figs. 1 and 2) with the radial velocity of the HeI streamer along the same line of sight, we note that for many of them the two velocities are quite comparable 
Table 3. Comparison of the radial velocities of the He I stars and the local ISM

\begin{tabular}{|ll|rl|c|}
\hline ID & \multicolumn{1}{|c|}{ Name } & $V_{\mathrm{R}}$ Star $\left(\mathrm{km} \mathrm{s}^{-1}\right)$ & $V_{\mathrm{R}}$ ISM $\left(\mathrm{km} \mathrm{s}^{-1}\right)$ \\
\hline \hline N1 & IRS 16NE & +1 & \pm 30 & +16 \\
N2 & IRS 16C & -24 & \pm 17 & -84 \\
N6 & HeI N2 & -97 & \pm 60 & -58 \\
N7 & IRS 34W & -175 & \pm 100 & -150 \\
\hline B2 & IRS 7E2 & +85 & \pm 130 & +70 \\
B3 & IRS 9W & +221 & \pm 55 & +309 \\
B6 & IRS 7W & -292 & \pm 45 & -250 \\
\hline
\end{tabular}

in amplitude, and with the same sign. The comparison is presented in Table 3 with the narrow-line stars in the upper part, and the broad-line stars in the lower part.

\section{Notes on individual He I stars}

In the following we discuss the peculiarities of some of the emission line profiles separated into the two classes presented in Figs. 1 and 2, and in Tables 1 and 2 .

\subsection{Narrow-line stars}

- [N3] IRS 16SW: This star shows a clear P Cygni profile with a $F W H M$ of the emission component of $258 \mathrm{~km} \mathrm{~s}^{-1}$. However, from all the stars of this class (Fig. 1) this one has the largest positive radial velocity of $354 \mathrm{~km} \mathrm{~s}^{-1}$. Ott et al. (1999) have been able to show from photometric observations that this star is a short-period variable, and therefore is probably a massive eclipsing binary. Consequently, the radial velocity plotted in Fig. 5 is not representative of the system itself, because of the contribution of the orbital velocity which could be as high as $677 \mathrm{kms}^{-1}$ from Ott et al. (1999). This might explain the reason for which the diagonal in Fig. 3 barely crosses the error box.

- [N5] IRS 33SE: Its He I line profile does not show a P Cygni profile. This star lies in the helium MiniSpiral, and the spectrum of the source before correction shows several ISM components, one of which falls where the absorption component should be. It was not possible in the correction of the stellar line profiles to recover this probable absorption component. Therefore, IRS 33SE might have a regular P Cygni profile which would imply a less positive radial velocity than displayed in Fig. 5. It would explain the discrepancy with the estimation of Eckart \& Genzel (1997) noted in Sect. 4.3, and shown in Fig. 3, as long as the estimation was based on the line of an element not present in the Mini-Spiral. It cannot be from $\mathrm{Br} \gamma$ for which the ISM emission strongly affects the stellar profiles (Morris \& Maillard 2000).

- [N6] HeI N2: A nearby star, IRS $16 \mathrm{CC}$, is reported as a helium star in Blum et al. (1996a), but by combining the BEAR data and the AO image as explained in Sect. 3.6, we find that the true helium star is in fact a fainter star, located $0.6^{\prime \prime}$ South and $0.3^{\prime \prime}$ East of IRS 16CC. This newly detected helium stars lies both in the wings of He I stars IRS $16 \mathrm{NE}$ and IRS $16 \mathrm{C}$ and in the Northern Arm of the ISM emission, which makes it difficult to identify. This star has the weakest $m_{K}$ in the narrow-line profile group (Table 2), with a value typical of the broad-line group.

- [N7] IRS 34W: This identification is reported in Krabbe et al. (1995) while Blum et al. (1996a) indicate simply IRS 34 . From the AO image there are effectively two stars of comparable brightness, IRS $34 \mathrm{E}$ and IRS $34 \mathrm{~W}, 0.4^{\prime \prime}$ to the South-West, within the BEAR box. The value of the continuum given in Table 1 and the magnitude in Table 2 is corrected for the contribution of IRS $34 \mathrm{E}$. With the $S / N$ ratio of the spectrum (Fig. 1) we cannot confirm a P Cygni profile for this line. This star has an $m_{K}$ value comparable to that of HeI N2. These two stars form a sub-group in the narrow-line group, which is discussed in Sect. 6.1.2.

\subsection{Broad-line stars}

- [B1] ID 180: This star is another of the newly detected helium stars. It falls in coincidence with a star for which the photometry is given in Ott et al. (1999);

- [B2] IRS 7E2: We propose this new identification for the helium star initially indicated as IRS 7E. From the AO image (Fig. 2) there are clearly two components, not noted before, that we defined as IRS 7E1 and IRS 7E2, separated by $0.37^{\prime \prime}$. IRS $7 \mathrm{E} 2$ is well centered within the BEAR box. The flux in Tables 1 and 2 is corrected correspondingly;

- [B5] IRS 13E3: This helium star was originally designated simply as IRS 13E (Krabbe et al. 1995; Blum et al. 1996a). In a later paper, IRS 13E1 is given as the helium star (Najarro et al. 1997a), whose photometry is given by Ott et al. (1999) along with the other component IRS 13E2, indicating two equally bright components $\left(m_{K}=10.26\right)$ separated by $\sim 0.14^{\prime \prime}$, which is the current limit of resolution of this work after deconvolution. By a local zoom of the AO image (Fig. 8), a fainter third component is seen, that we call IRS 13E3. By applying the procedure described in Sect. 3.6 the bright BEAR pixel is projected onto the AO image. The flux in the $2.058 \mu \mathrm{m}$ line could come from either $13 \mathrm{E} 2$ or $13 \mathrm{E} 3$. The helium source in this complex is a broad-line star, therefore with a relatively weak continuum. As the faintest component, IRS 13E3 is the most plausible candidate. The $K$ magnitude in Table 2 corrected accordingly $\left(m_{K}=11.73\right)$ provides a value in agreement with the magnitude of the stars of the same group, which would not be the case with IRS $13 \mathrm{E} 2$. Of course, only diffraction-limited spectro-imaging can unambiguously confirm this identification. Otherwise, the line profile presented in Fig. 2 is one of those where 


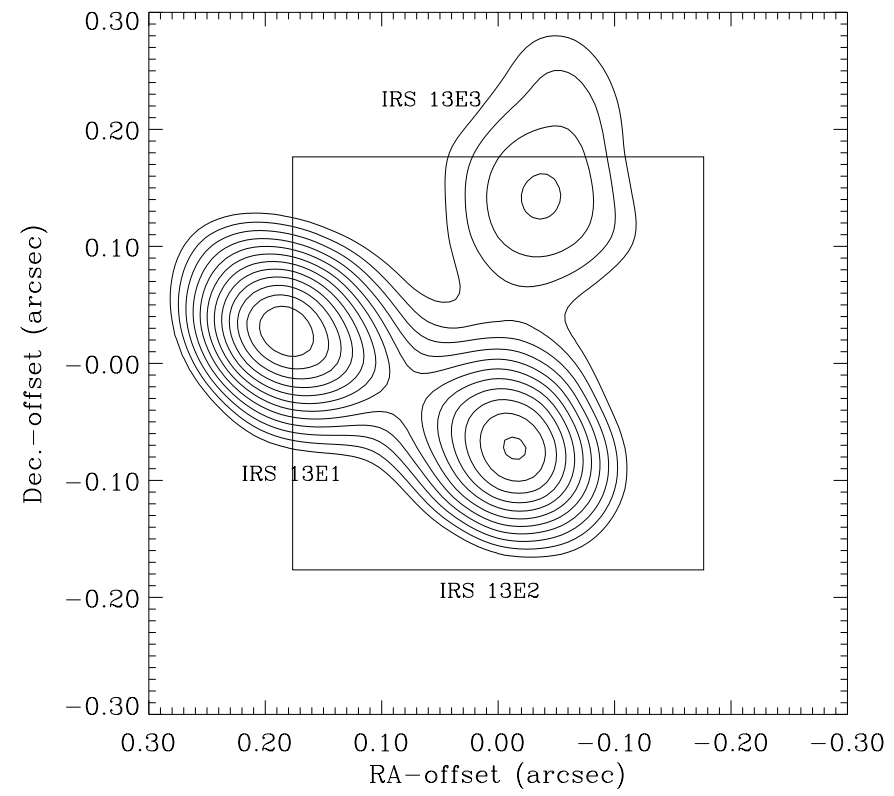

Fig. 8. Zoom of the $K$-band adaptive optics image in contour on the IRS 13E complex showing the 3 stellar components. The square box represents the position of the maximum intensity BEAR pixel at the IRS 13 position in the line cube, projected on the AO image. IRS 13E3 is considered to be the helium star (see Sect. 5.2)

an important correction to remove the ISM contribution, made of several components, was needed. This results in a very flat top;

- [B7] AF: This object has been long recognized as a helium star and has been the subject of detailed studies, in particular by Najarro et al. (1994). However, in the broad-line class of objects this star is an exception, with a continuum brighter than all the members of this group (Tables 1 and 2). The intensity of the emission relative to the continuum (Fig. 2) is comparable to the intensity of other stars of this group, but intrinsically, the line emission is the most intense of all the helium stars. From the AO image, there is a cluster of 5 sources with one dominant source. The BEAR aperture contains this source plus two of the fainter sources. From Ott et al. (1999), this star presents a strong index of variability, the origin of which is not determined. The possibility that the variability is periodic should be checked, as that might indicate a compact binary star which could explain the peculiarity of this star. At any rate, AF cannot be considered as typical for all the other He I stars, as implied by Najarro et al. (1994, 1997a);

- [B9] HeI N3: This is the third new helium star. Its corresponding object in the AO image was found with a $K$ magnitude (Table 2 ) in agreement with the mean value found for the broad-line stars. The line shows the largest redshift among all these stars. The error bar is large since the emission is relatively weak. The $S / N$ ratio is not high enough to show a $\mathrm{P}$ Cygni profile.

\section{Discussion}

The main results from this study of the helium emissionline stars in the central pc of the Galactic Center can be summarized as follows:

1) Sixteen fully resolved P Cygni emission line profiles, purely of stellar origin, are extracted;

2) They divide into two distinct classes, with narrow and broad-line profiles;

3 ) The stars in each group have a comparable $K$ magnitude but the two groups show a mean difference of $\sim 2 \mathrm{mag}$

4) The spatial distribution of the two groups is different. The narrow-line objects are all arranged in a central cluster, while the other class are dispersed in a ring beyond a radius of $\simeq 0.3 \mathrm{pc}$ from $\operatorname{SgrA}^{\star}$;

5) ISM emission of helium streamers which follow the Mini-Spiral is discovered. The radial velocity of these flows seen along the line of sight to a large fraction of the helium stars is comparable to the radial velocity of the underlying star.

Hence, we must examine all the observed findings to determine what they tell us about the nature and the formation of these stars.

\subsection{The stellar type of the Hel stars in the inner GC}

The P Cygni lineshape for the helium emission of most of the stars we observed indicates that all of them are hot stars which possess an extended atmosphere in rapid expansion. However, the two different classes of line profile associated with the remarkable anti-correlation with the continuum brightness call for two different types of hot, helium-rich stars. IRS $16 \mathrm{C}$ and IRS $7 \mathrm{~W}$ are typical examples of each class. The differences cannot be ascribed to orientation, such as an equatorial gaseous envelope seen edge-on or pole-on, as has been proposed to explain the two different types of emission line profiles in Be stars. Over the set of sources a continuity in the linewidths would be observed, with a double peak in some cases, while a single line is always observed, but with two radically different linewidths. These profiles are clearly suggestive in all cases of strong wind outflows. To which stellar types do these different profiles belong? Do they correspond to massive, hot stars but at two different stages of evolution?

\subsubsection{The stellar types of massive, hot stars}

The question of the stellar type of the He I stars has already been examined by all the authors who have previously studied this stellar population. Note that they all make the preliminary remark that the study of the earlytype GC stars forces a revision of the usual stellar classification criteria, generally based on visible spectra, to find their translation in the near infrared. This has triggered various spectroscopic studies of hot stars conducted in the $K$ band, by Hanson \& Conti (1994), Blum et al. (1995b), 
Tamblyn et al. (1996), Morris et al. (1996), Hanson et al. (1996), and Figer et al. (1997). It turns out that the hot stars which exhibit the HeI $2.058 \mu \mathrm{m}$ line in emission belong to a large variety of spectral types, from normal Oe, Be stars and B supergiants (Hanson et al. 1996) to sub-types of peculiar, luminous stars which are the $\mathrm{B}[\mathrm{e}]$, LBV (Luminous Blue Variables), WR stars, and intermediate types like ON, Ofpe, Ofpe/WN9, undergoing a strong mass loss. Discussion of these various stellar types are included in Libonate et al. (1995) and in Tamblyn et al. (1996) who discuss the helium stars in the GC. Tracks of evolution of massive stars, depending on the initial mass and the metallicity, have been proposed by Meynet et al. (1994). A typical sequence for massive stars, for example of $60 M_{\odot}$, is: O, Blue Supergiant, LBV and WR, as they evolve to becoming SNs. The WR stars represent the final stage of evolution of massive $\mathrm{O}$ stars of initial mass $\geq 40 M_{\odot}$. Two main sub-classes, the $\mathrm{WN}$ and the $\mathrm{WC}$ stars, depend on their phase of nucleosynthesis, the WC stars being the most evolved of the WR stars (Abbott \& Conti 1987).

The $K$-band atlas of Figer et al. (1997) is devoted to the WR stars. These authors conclude that from this spectral range it is not easy to distinguish between individual sub-types, in particular for WC stars since their $K$ spectra tend to be quite similar. Regarding the $2.058 \mu \mathrm{m}$ line, they show that this line is present in late $\mathrm{WN}$-types and otherwise, is particularly prominent in WC9 types. That is partially confirmed by Tamblyn et al. (1996), who also mention a strong $2.058 \mu \mathrm{m}$ line with a $\mathrm{P}$ Cygni profile for the WN8 and the LBV members of their star sample. They also note the line in simple emission, for the few late ON-type and early B-type supergiants they observe. Hanson et al. (1996) detect the line in emission for $\mathrm{OeV}$ et $\mathrm{BeV}$ stars but with a complex profile, and otherwise in supergiant B1 stars. Finally, it seems difficult to draw very clear conclusions since all these intermediate classes, such as LBV, ON or Ofpe, represent very rare groups of stars. For example, from a review of the statistics of LBVs and related stars by Parker (1997), there are only 5 confirmed LBV stars in the Milky Way disk, including the two famous examples P Cygni itself and $\eta$ Car, and only 26 more within 8 nearby galaxies, including the LMC and SMC. However, Parker notes that there are more candidates if more "liberal definitions" are applied, which means that it is not possible to generally assign a strict spectral type to these stars, in particular from a study carried out only within a limited spectral range.

The WR stars are known for extremely broad emission lines (Abbott \& Conti 1987), for which the values of $\sim 1000 \mathrm{~km} \mathrm{~s}^{-1}$ and more are typical, comparable to the FWHM reported in Fig. 2. On the other hand, the line profile observed for He I in the LBV star P Cygni is quite comparable to the $2.058 \mu \mathrm{m}$ line profiles we call narrow-line profiles (Fig. 1). High resolution observations of the infrared emission lines of P Cygni by Najarro et al. (1997b) give lines with profiles having widths fitted by a model with a terminal velocity of $185 \mathrm{~km} \mathrm{~s}^{-1}$ and a $T_{\text {eff }}$ of
$18100 \mathrm{~K}$. The existence of an extended helium envelope for P Cygni is given by the interferometric observations of Vakili et al. (1997) in the HeI $6678 \AA$ line. They estimate a photospheric radius $R_{\star}=76 \pm 15 R_{\odot}$ and an extent of the helium envelope of $12.5 R_{\star}$.

\subsubsection{The Hel stars as LBV and WR-type stars}

Thus, from only the consideration of the two distinct types of He I line profiles, the helium stars with narrow-line profiles should be most closely related to LBV-type stars, while the other group to WR-type stars, without trying to be more specific. Tamblyn et al. (1996), who had already noticed few HeI stars in the inner pc with narrower line profiles, have considered whether these sources might be LBVs. They contest this hypothesis on the basis that LBV stars are a too brief phase of stellar evolution, which explains their rarity, and that these stars are not hot enough to be efficiently detected in the $2.058 \mu \mathrm{m}$ emission line. This conclusion is based on the observation of only two galactic LBV stars, of which effectively only one (P Cygni) shows clearly the $2.058 \mu \mathrm{m}$ line in emission, whereas the other has a poor $S / N$ ratio. Thus, these arguments are not very convincing.

One of the general parameters which distinguishes these two types of stars is their range of effective temperature $\left(T_{\text {eff }}\right)$. Hence, a range of $T_{\text {eff }}$ is reported from observations of LBVs (Crowther 1997), from $8000 \mathrm{~K}$ to $\sim 25000 \mathrm{~K}$, while for WRs the range is definitely beyond, from 30000 to $\sim 90000 \mathrm{~K}$ (van der Hucht et al. 1991). For all these stars, the $2 \mu \mathrm{m}$ region is far from the maximum of emission. However, although hotter, the WR stars are intrinsically dim among massive stars, because of their relatively small photosphere radii. WC stars are typically $10^{5} L_{\odot}$. These can be contrasted to LBV stars, which sit near the Humphreys-Davidson limit, typically above $10^{6} L_{\odot}$. This fundamental difference is attributable to the copious mass loss experienced by the most massive $\mathrm{O}$ stars $\left(M \simeq 120 M_{\odot}\right)$ which will end their lives with a mass between 5 and $10 M_{\odot}$, when they are WR stars. Thus, this distinction between LBV and WR fits also with the observed difference in $K$ magnitude between the two groups of He I stars (point \# 3 of the summary).

The particular cases of IRS $34 \mathrm{~W}$ and HeI N2 must be discussed within the framework of this classification. These two stars belong to the LBV-type group from their line profile, but with an $m_{K}$ typical of the other group (Table 2). The few galactic LBVs studied in detail are known to be characterized by giant eruptions which are followed by dust obscuration. From the reconstructed light curve of $\eta$ Car (Humphreys et al. 1999), the maximum obscuration lasted about $\sim 40$ years since the last eruption. From its pre-outburst level it had undergone a 4-mag visual extinction. These two LBV-type star candidates might be in such a phase.

In conclusion, we propose that the class of narrowline stars consists of LBVs or related stars in the 10000 
to $20000 \mathrm{~K}$ range of $T_{\text {eff }}$, while the second class consists of much hotter stars $(\geq 30000 \mathrm{~K})$, late-type WR stars, predominantly in the WC9 stage, according to the conclusions of Figer et al. (1997) from their $K$-band atlas of WR stars. The latter is also in agreement with the classification properly made for one helium star (BSD WC9) by Blum et al. (1995a). However, from these considerations only, the mere distinction between luminous blue stars and LBVs is not really possible. Strictly speaking, variability should be established to identify an LBV. That is only suggested by the status of IRS $34 \mathrm{~W}$ and HeI N2 compared to the other sources of the group. While WR stars seem the most likely for one group, only the proximity in evolution pleads in favor of LBV-related status for the other group, which is discussed in the next section, in relation to their spatial distribution (point \# 4 of the summary).

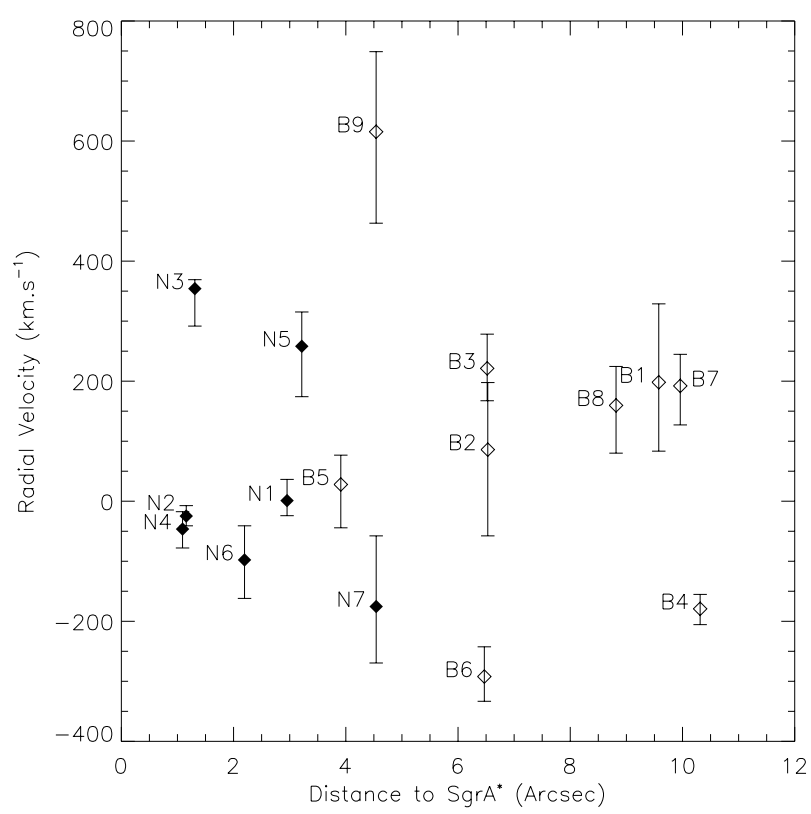

Fig. 9. Observed radial velocity of the He I stars as a function of projected distance from $\mathrm{SgrA}^{\star}$. Symbols have the same meaning as in Fig. 3

\subsection{Spatial distribution of the Hel star and their formation}

Is the above classification the result of a sequence of evolution, since LBVs are precursors of WR stars? A parameter to take into account is the initial mass of the progenitors. An important conclusion of the evolutionary tracks of Meynet et al. (1994) is that, for the most massive stars $-\geq 120 M_{\odot}-$ the LBV stage is avoided to go directly from $\mathrm{O}$ or Of to late WN, then WC and SN. Therefore, to be of the same age, the LBV-type stars must have originated from massive $\mathrm{O}$ stars in the range 40 to $120 \mathrm{M}_{\odot}$ while the WR group should be originating from stars of initial mass $\geq 120 M_{\odot}$, which thus reached directly the WR stage where they are currently observed.

The placing in Fig. 9 of the radial velocities as a function of the projected distance of the sources from $\mathrm{SgrA}^{\star}$ is another way of showing the two groups clearly distributed in two concentric volumes around $\mathrm{SgrA}^{\star}$, with approximately equal velocity distribution. If we adhere to the coeval formation scenario, then the difference of spatial distribution of the two types of HeI stars, presented in Figs. 4 and 9, must be explained in this context, and we ask if that should be the signature of the star formation process.

\subsubsection{The WR-type star ring}

Figures 4 and 9 suggest that there are no orbits of WR stars in planes perpendicular to the plane of the sky, since we do not see any of them projected close to $\operatorname{Sgr} A^{\star}$. Also, we measure radial velocities for these stars between $-300 \mathrm{kms}^{-1}$ and $+600 \mathrm{~km} \mathrm{~s}^{-1}$ (Fig. 9). So, the orbits cannot be in the plane of the sky either, or all the $V_{\mathrm{R}} \mathrm{s}$ would be close to 0 . Thus, in agreement with Genzel et al. (2000), we can conclude that the orbits of these stars reside roughly in a disk inclined by $40^{\circ}$ off the plane of the sky, with quasi-circular orbits, which fits with their distribution in a ring of $\sim 0.2 \mathrm{pc}$ radial width, centered on $\mathrm{SgrA}^{\star}$. The sign of the radial velocities (Fig. 5) is consistent with the orbits described in a general clockwise sense, as derived from proper motions studies (Genzel et al. 2000). However, these authors note that "the fit of the best Keplerian disk model to the He I star velocities is poor".

\subsubsection{The LBV-type star cluster}

The stars with narrow-line profiles are grouped in a cluster close to $\mathrm{SgrA}^{\star}$. If circular orbits are also assumed for these stars the orbital radii must be small in order for them to appear as a cluster in projection. For the closest He I stars to $\operatorname{SgrA}^{\star}$ (N2, N3 and N4 in Fig. 9, within a radius of $0.06 \mathrm{pc}$ ) the orbital velocity should be of the order of $600 \mathrm{~km} \mathrm{~s}^{-1}$. The largest measured radial velocity is $354 \mathrm{~km} \mathrm{~s}^{-1}$ for N3, so pure Keplerian motions are possible for these stars. With such velocities on a small orbital radius, a proper motion becomes detectable within a few years, as reported by Ghez et al. (1998) and Genzel et al. (2000). Then, for star N2 (IRS 16C) for which a $V_{\mathrm{R}}$ of only $-24 \mathrm{~km} \mathrm{~s}^{-1}$ is measured (Fig. 9) the modulus of the projected proper motion velocity is $480 \mathrm{~km} \mathrm{~s}^{-1}$ from the measurements of Genzel et al. (2000). With the correction for projection an orbital velocity consistent with circularity is possible. At least, from the observations, very elongated orbits are excluded.

\subsubsection{Tentative conclusions}

The massive, hot stars are concentrated in the central pc around $\operatorname{SgrA}^{\star}$. To remain concentrated in that position 
requires that their orbits are dominated by the gravitational field of the central Black Hole. From the conclusions of Genzel et al. (2000), the overall rotation of the He I cluster is a remnant of the original angular momentum pattern in the interstellar cloud from which these stars formed. Indeed, they may have formed together in a gaseous disk orbiting the central black hole, less than $\sim 5$ Myr ago from the life-time of WR stars. However, our observations imply that the stars differentiated according to their distance from the central Black Hole, into two star groups, distinguished by their initial mass, with more massive stars forming at a larger distance. This could be obtained, actually, if the initial disk was formed of two separate rings, one with a mean radius of $\sim 0.04 \mathrm{pc}$, the other one of $\sim 0.3 \mathrm{pc}$. If in addition, the SgrA ${ }^{\star}$ cluster (Genzel et al. 1997; Ghez et al. 1998) is considered, it continues the trend toward smaller masses located inward. These stars form a separate third group of main sequence, early-type stars (Genzel et al. 2000). Then, all these early-type stars may have formed in the same star formation event from a gaseous disk around the central mass, but with annular structures, probably caused by tidal forces, which remain to be explained.

\subsection{The link between Hel streamers and Hel stars}

As very hot stars and with helium dominating their chemical composition, WR stars are sources of a strong ionizing flux (Schmutz et al. 1992), thus implying the presence of a hard UV field in the inner pc. This is consistent with the presence of the helium Mini-Spiral (Fig. 6) we detect in emission in the near infrared, since hard ionizing photons are required to excite this line in the ISM. However, this UV field cannot penetrate very deep into the flows, because it will be blocked by the dust. Therefore, the helium streamers should delineate the inner regions of the gaseous Mini-Spiral. That is likely the reason for which the helium emission map (Fig. 6) appears simpler than in $\operatorname{Br} \gamma$ (Morris $\&$ Maillard 2000), and why the linewidth is relatively narrow $\left(<70 \mathrm{~km} \mathrm{~s}^{-1}\right)$. However, as mentioned in Sect. 4.6, the same main emission structures are seen in He I and in $\mathrm{Br} \gamma$, as well as in $\mathrm{Ne}^{+}$(Lacy et al. 1991). The ISM helium data cube indicates that the gas is distributed in several flows. But these gaseous orbits do not follow the earlytype star distribution which has been described above. This implies that the overall kinematics of the gas and these stars are different. However, we noticed a similarity of radial velocity (point \# 5 of the summary) between the He I stars and the He I streamers, along the same line of sight (Table 3). This suggests that the kinematics of the streamers, within a radius of $\sim 0.5 \mathrm{pc}$ from $\mathrm{SgrA}^{\star}$, become mainly dominated by the gravitational field of the central Black Hole. At further distances reached by the Mini-Spiral, the field is more complex, with the contribution of all the other stars, which are essentially latetype stars. From the photometric survey of the central 5 parsecs of the Galaxy by Blum et al. (1996a) it can be estimated that $\sim 80 \%$ of the known stellar population is comprised of stars identified as late-type giants and supergiants (e.g. IRS 7) by their CO absorption (Blum et al. 1996b). These K, M and AGB stars which correspond to a mass range of 2-8 $M_{\odot}$ (except for the few supergiants) are likely much older $\left(\sim 10^{8}-310^{9} \mathrm{yr}\right)$ than the current helium stars. They must have been produced in a totally different star formation event. They are stars with important mass loss, but not of helium-enriched material. Hence, this suggests that the observed concentration of He I stars should be the major source of enhancement of the helium abundance in the inner GC. From the average mass loss rate reported for $\mathrm{LBV}$ and $\mathrm{WR}$ stars, and with the presence of around 20 of such stars, the total mass loss rate, only from all these stars in the central pc, could be estimated at $\sim 210^{-3} M_{\odot} /$ yr. The dynamical time for the gas in the streamers of SgrA West is about $10^{4} \mathrm{yr}$, so on this time scale, $\sim 20 M_{\odot}$ of He-enriched stellar material might be mingling with the infalling matter in the streamers. The observed similarity of velocity, in amplitude and sign, of the HeI stars and of the flows suggests a close link in the formation of both. The helium-rich Mini-Spiral might be the precursor of the disk in which will form the next, massive, hot star generation.

\section{Conclusions and perspectives}

New results on the population of He I stars in the inner region of the Galactic Center have been obtained with the firm indication of two classes of massive hot stars, which suggest a formation in a disk of gas around SgrA*. This analysis is based on a single line, the He I $2.058 \mu \mathrm{m}$ line. Of course, while that is not sufficient to establish a complete spectral classification, one can nonetheless consider that this study represents a necessary initial selection test, since it has been shown that several stars in previous studies were wrongly considered as HeI stars. By consequence, it illustrates also the risk of false detections in the search for emission-line stars toward the inner Galaxy simply by using narrow-band photometry centered on the He I $2.058 \mu \mathrm{m}$ line, or other near-infrared emission lines. The importance of high spectral resolution combined with high spatial resolution is paramount for distinguishing stellar and interstellar emission. Similar data obtained with BEAR already exists on $\operatorname{Br} \gamma$ (Morris \& Maillard 2000). With the same effort for separating the stellar and the interstellar component, the $\mathrm{Br} \gamma$ line profile for the 16 confirmed He I stars should be retrieved, making another test on this stellar population. The next goal is a larger spectral coverage to complete the spectral criteria. That has already been done by several previous works, but always at medium resolution and with slit spectrometers, for which the source confusion is not easily controlled. Only a slitless technique like that employed by BEAR makes this control possible. Also, similar studies on other critical lines must be conducted. However, the next major step, instead of spectro-imaging at seeing-limited resolution as we have presented, and tried to improve by combining with AO imaging, will be infrared spectro-imaging at the 
diffraction-limited spatial resolution of a large telescope. That is the only way to detect more sources in order to remove all the identification ambiguities. As illustrated here, this should be combined with a spectral resolution of at least 5000, which is not an easy goal.

Finally, it would be important to conduct similar studies in other stellar clusters like the "Arches" and "Quintuplet" clusters (Figer et al. 1999a, 1999b) where the identification of LBV stars has also been proposed, notably the Pistol star (Figer et al. 1999c), to determine comparatively the conditions of evolution of young compact clusters at Galactocentric distances well beyond the central pc of the Milky Way.

Acknowledgements. We would like to thank warmly Doug Simons (now at Gemini) who participated actively in the early development of BEAR and designed the camera Redeye, which is used on the instrument. He was part of the observing run when the data were acquired, and of a preliminary run the year before. We are also grateful to the CFHT staff for the technical support of BEAR and of the data acquisition program which is associated.

\section{References}

Abbott, D. C., \& Conti, P. S. 1987, ARA\&A, 25, 113

Allen, D. A., Hyland, A. R., \& Hillier, D. J. 1990, MNRAS, 244,706

Blum, R. D., Sellgren, K., \& DePoy, D. L. 1995a, ApJ, 440, L17

Blum, R. D., DePoy, D. L., \& Sellgren, K. 1995b, ApJ, 441, 603

Blum, R. D., Sellgren, K., \& DePoy, D. L. 1996a, ApJ, 470, 864

Blum, R. D., Sellgren, K., \& DePoy, D. L. 1996b, AJ, 112, 1988

Castor, J. I., \& Lamers, H. J. G. L. M. 1979, ApJS, 39, 481

Crowther, P. A. 1997, in Luminous Blue Variables: Massive Stars in Transition, ed. A. Nota, \& H. Lamers, ASP Conf. Ser., 120, 51

Doyon, R., Nadeau, D., Vallée, P., et al. 1998, in Infrared Astronomical Instrumentation, ed. A. M. Fowler, Proc. SPIE, 3354, 760

Elias, J. H., Frogel, J. A., Mathews, K., \& Neugebauer, G. 1992, AJ, 87, 1029

Eckart, A., \& Genzel, R. 1997, MNRAS, 284, 576

Figer, D. F., McLean, I. S., \& Najarro, F. 1997, ApJ, 486, 420

Figer, D. F., Mc Lean, I. S., \& Morris, M. 1999a, ApJ, 514, 202

Figer, D. F., Kim, S. S., Morris, M., et al. 1999b, ApJ, 525, 750

Figer, D. F., Morris, M., Geballe, T. R., et al. 1999c, ApJ, 525, 759

Geballe, T. R., Krisciunas, K., Bailey, J. A., \& Wade, R. 1991, ApJ, 370, L73

Genzel, R., Thatte, N., Krabbe, A., Kroker, H., \& TacconiGarman, L. E. 1996, ApJ, 472, 153

Genzel, R., Eckart, A., Ott, T., \& Eisenhauer, F. 1997, MNRAS, 291, 219

Genzel, R., Pichon, C., Eckart, A., Gerhard, O. E., \& Ott, T. 2000, MNRAS, 317, 348

Ghez, A. M., Klein, B. L., Morris, M., \& Becklin, E. E. 1998, ApJ, 509, 678
Hall, D. N. B, Kleinman, S. G., \& Scoville, N. Z. 1982, ApJ, 260, L53

Hanson, M. M., \& Conti, P. S. 1994, ApJ, 423, L139

Hanson, M. M., Conti, P. S., \& Rieke, M. J. 1996, ApJS, 107, 281

Humphreys, R. M., Davidson, K., \& Smith, N. 1999, PASP, 111,1124

van der Hucht, K. A. 1991, in Wolf-Rayet Stars and Interrelations with Other Massive Stars in Galaxies, IAU Symp. 143, ed. K. A. van der Hucht, \& B. Hidayat, 19

Krabbe, A., Genzel, R., Drapatz, S., \& Totaciuc, V. 1991, ApJ, 382, L19

Krabbe, A., Genzel, R., Eckart, A., et al. 1995, ApJ, 447, L95

Lacy, J. H., Achtermann, J. M., \& Serabyn, E. 1991, ApJ, 380, L71

Lai, O., Véran, J. P., Rigaut, F., et al. 1997, in Optical Telescopes of Today and Tomorrow, ed. A. L. Ardeberg, Proc. SPIE, 2871, 859

Libonate, S., Pipher, J. L., Forrest, W. J., \& Ashby, M. L. N. 1995, ApJ, 439, 202

Lutz, D. 1998, in The Universe as seen by ISO, ed. P. Cox, \& M. F. Kessler, ESA Pub., SP-427, 623

Maillard, J. P., \& Michel, G. 1982, in Instrumentation for Astronomy with Large Telescopes, IAU Coll. No. 92, ed. C. M. Humphries (D. Reidel, Pub.), 213

Maillard, J. P. 1995, in Tridimensional Optical Spectroscopic Methods in Astrophysics, IAU Col. 149, ed. G. Comte, \& M. Marcelin, ASP Conf. Ser., 71, 316

Maillard, J. P. 2000, in Imaging the Universe in 3 Dimensions, ed. E. van Breughel, \& J. Bland-Hawthorn, ASP Conf. Ser., 195, 185, available at http://www . aao.gov.au/local/www/jbh/3D/

Menten, K. M., Reid, M. J., Eckart, A., \& Genzel, G. 1997, ApJ, 475, L111

Meynet, G., Maeder, A., Schaller, D., \& Charbonnel, C. 1994, A\&AS, 103, 97

Morris, M. 1985, in Mass Loss from Red Giants, ed. M. Morris, \& B. Zuckerman (Dordrecht: Reidel), 124

Morris, M. 1993, ApJ, 408, 496

Morris, M., \& Maillard, J. P. 2000, in Imaging the Universe in 3 Dimensions, ed. E. van Breughel, \& J. Bland-Hawthorn, ASP Conf. Ser., 195, 196, available at http://www . aao.gov.au/local/www/jbh/3D/

Morris, P. W., Eenens, P. R. J., Hanson, M. M., Conti, P. S., \& Blum, R. D. 1996, ApJ, 470, 597

Najarro, F., Hillier, D. J., et al. 1994, A\&A, 285, 573

Najarro, F., Krabbe, A., Genzel, R., et al. 1997a, A\&A, 325, 700

Najarro, F., Hillier, D. J., Lamers, H. J. G. L. M, et al. 1997b, in Luminous Blue Variables: Massive Stars in Transition, ed. A. Nota, \& H. Lamers, ASP Conf. Ser., 120, 105

Ott, T., Eckart, A., \& Genzel, R. 1999, ApJ, 523, 248

Parker, J. W. 1997, in Luminous Blue Variables: Massive Stars in Transition, ed. A. Nota, \& H. Lamers, ASP Conf. Ser., 120,368

Reid, M. J. 1993, ARA\&A, 31, 345

Schmutz, W., Leitherer, C., \& Gruenwald, R. 1992, PASP, 104, 1164

Simons, D. A., Clark, C. C., Smith, S., et al. 1994, in Instrumentation in Astronomy VIII, Proc. SPIE, 2198, 185

Tamblyn, P., Rieke, G. H., Close, L. M., et al. 1996, ApJ, 456, 206 